\title{
Antimicrobial Resistance and Genotyping of Bacteria Isolated from Urinary Tract Infection in Children in an Iranian Referral Hospital
}

This article was published in the following Dove Press journal: Infection and Drug Resistance

\author{
Setareh Mamishi ${ }^{1,2}$ \\ Zohreh Shalchi ${ }^{3}$ \\ Shima Mahmoudi ${ }^{1}$ \\ Reihaneh Hosseinpour Sadeghi ${ }^{1}$ \\ Mohammad Taghi Haghi Ashtiani ${ }^{4}$ \\ Babak Pourakbari' \\ 'Pediatric Infectious Diseases Research \\ Center, Tehran University of Medical \\ Sciences, Tehran, Iran; ${ }^{2}$ Department of \\ Infectious Diseases, Pediatrics Center of \\ Excellence, Children's Medical Center, \\ Tehran University of Medical Sciences, \\ Tehran, Iran; ${ }^{3}$ Department of Pediatrics, \\ Hamadan University of Medical Sciences, \\ Hamadan, Iran; ${ }^{4}$ Department of \\ Pathology, School of Medicine, Tehran \\ University of Medical, Sciences, Tehran, \\ Iran
}

Introduction: Urinary tract infection (UTI) is one of the most common bacterial infections in childhood, and the increasing rate of antibiotic resistance to the commonly prescribed antimicrobial agents against it has become a major concern. The aim of this study was to determine the antibiotic resistance and genotyping of bacteria isolated from urine cultures in patients referred to the Children's Medical Center, Tehran, Iran.

Methods: During the 1-year period, antimicrobial susceptibility profiles of strains isolated from patients with UTI were determined. Typing of the isolates causing nosocomial infections was performed by random amplified polymorphic DNA (RAPD) analysis, and the results were analyzed by Gelcompar II software.

Results: In this study, 203 children (130 girls and 73 boys) were included. The patients' age ranged from 1 day to 16 years (IQR average $=4$ months to 4 years). The most frequent isolated organisms were Escherichia coli (118 isolates, 58\%), followed by Klebsiella pneumoniae (30 isolates, $15 \%$ ). Sixty-two strains (18 strains of E. coli, 13 strains of $K$. pneumoniae, 11 strains of Enterococcus faecium, and five strains of Burkholderia cepacia complex) had criteria of nosocomial infection. A high resistance rate to trimethoprim-sulfamethoxazole $(69 \%)$ and cefotaxime $(60 \%)$ was reported in $E$. coli and $K$. pneumoniae strains, respectively. Pseudomonas aeruginosa strains showed high sensitivity to amikacin (100\%). All E. faecium strains were susceptible to trimethoprim-sulfamethoxazole (100\%), and $23 \%$ of the strains were resistant to vancomycin. The analysis of RAPD-typing revealed the presence of three clusters in E. coli, two clusters in E. faecium, and one clone in K. pneumoniae. Besides, four out of five isolates of $B$. cepacia complex had more than $90 \%$ genetic similarity.

Conclusion: The most frequent isolated pathogen was $E$. coli, and an increasing rate of antibiotic resistance to the commonly prescribed antimicrobial agents such as trimethoprim/ sulfamethoxazole and cephalosporins was observed. Moreover, the results of this study showed the presence of clones with $\geq 80 \%$ similarity in E. coli, K. pneumoniae, E. faecium, and B. cepacia complex isolates; therefore, the transmission of nosocomial infections from one patient to another or one ward to another is probable.

Keywords: urinary tract infection, antibiotic resistance pattern, genotyping

\section{Introduction}

Urinary tract infections (UTIs) are one of the most common bacterial infections in children aged $<2$ years $^{1}$ and considered as a main cause of hospitalization in childhood. $^{2}$ The incidence rate of UTIs is up to $7 \%$ among febrile infants with no other recognizable causes. ${ }^{3}$ Escherichia coli has been known as the most common isolated organism in pediatric patients with UTI with a prevalence of $80-90 \%, 4,5$
Correspondence: Babak Pourakbari Pediatric Infectious Diseases Research Center, Children Medical Center Hospital, School of Medicine, Tehran University of Medical Sciences, No. 62, Gharib St., Keshavarz Blvd, Tehran, Iran Tel/Fax +98021 66428996

Email pourakbari@tums.ac.ir 
followed by others such as Enterococcus species (spp.), Enterobacter spp., Pseudomonas aeruginosa, Klebsiella pneumoniae, Proteus mirabilis, and Staphylococcus spp. ${ }^{5,6}$ In the preantibiotic era, UTI caused a mortality rate as high as $20 \%{ }^{7}$ The early treatment of UTI decreases the rate of morbidity; ${ }^{2}$ however, the increasing rate of antibiotic resistance to the commonly prescribed antimicrobial agents such as trimethoprim/sulfamethoxazole, cephalosporins, and fluoroquinolones ${ }^{8}$ has become a major concern. ${ }^{9}$ Hence, it is crucial to know the frequency of uropathogens and antimicrobial resistance in pediatrics. ${ }^{10}$ Prompt and efficient antimicrobial therapies for UTIs are vital for an appropriate outcome and prevention of long-term morbidity associated with renal scarring such as hypertension, development of chronic kidney disease, and ultimately the need of renal transplantation. ${ }^{11}$ The aim of this study was to determine the antibiotic resistance pattern and genotyping of strains isolated from pediatric patients with UTI.

\section{Materials and Methods}

This study was conducted in accordance with the Declaration of Helsinki and approved by the Ethics Committee of Tehran University of Medical Sciences, Tehran, Iran (IR.TUMS.CHMCREC.1397.47).

This study was performed over a period of 1 year, from March 2018 to February 2019 in he Children's Medical Center, an Iranian referral pediatrics hospital in Tehran, Iran. The inclusion criteria were patients having a history of fever $>38^{\circ} \mathrm{C}$ or symptoms of UTIs including dysuria, frequency, urgency, hesitancy, small volume voids, or lower abdominal pain. ${ }^{9}$ UTI was defined in infants and children as growth of a single pathogen of $>10^{5}$ colony forming units/mL by properly collected urine specimen (suprapubic aspiration, transurethral catheterization, or mid-stream urine) in febrile children or the ones who had other urinary symptoms. ${ }^{12}$ Bacteria identification was performed using Gram staining (for bacterial morphology) and differential biochemical tests. Identification of organisms was carried out using conventional biochemical methods such as catalase, oxidase, sugar fermentation, methyl red/Voges-Proskauer (MR/VP) test, growth on Kliger's Iron Agar (KIA), growth on Bile-Esculin Agar and $6.5 \% \mathrm{NaCl}$ media, and motility test. ${ }^{13}$

All isolated bacteria were checked for their susceptibility towards different antibiotics by disk diffusion method based on Clinical Laboratory Standard Institute (CLSI) guidelines, 2019. ${ }^{14}$ The list of antibiotic discs used for gram-negative bacteria were: nitrofurantoin (300 $\mu \mathrm{g})$, trimethoprim-sulfamethoxazole $(1.25 / 23.75 \mu \mathrm{g})$, cefepime $(30 \mu \mathrm{g})$, cefotaxime $(30 \mu \mathrm{g})$, imipenem $(10 \mu \mathrm{g})$, amikacin $(30 \mu \mathrm{g})$, piperacillin/tazobactam (100-10 g), ceftazidime $(30 \mu \mathrm{g})$, and gentamicin $(10 \mu \mathrm{g})$. Disks used for Gram-positive bacteria were gentamicin $(10 \mu \mathrm{g})$, penicillin (30 $\mu \mathrm{g})$, vancomycin $(30 \mu \mathrm{g})$, nitrofurantoin $(300 \mu \mathrm{g})$, Trimethoprim-sulfamethoxazole $(1.25 / 23.75 \mu \mathrm{g})$, piperacillin/tazobactam (100-10 g), imipenem $(10 \mu \mathrm{g})$, meticillin $(30 \mu \mathrm{g})$, and ampicillin $(10 \mu \mathrm{g})$. High-level resistance to gentamicin was also determined using a $120 \mu \mathrm{g}$ gentamicin disk. The minimal inhibitory concentration of vancomycin was determined by E-test methods. All disks were prepared by the Mast Group Ltd., Merseyside, UK. E. coli ATCC 25922, Pseudomonas aeruginosa ATCC 27853, and Staphylococcus aureus ATCC 25923 were used as control strains. All recovered strains which had caused nosocomial infections were typed by Random Amplified Polymorphic DNA PCR (RAPD-PCR) in a mixture consisting of $2.5 \mu \mathrm{L}$ of the PCR buffer (10-times concentrated), $0.75 \mu \mathrm{L}$ of $\mathrm{MgCl}_{2}$ (final concentration $250 \mu \mathrm{M}$ ), $0.6 \mu \mathrm{L}$ of dNTPs (Fermentas, Vilnius, Lithuania, final concentration $3 \mathrm{mM}$ ), $1 \mu \mathrm{L}$ of primer (5'- AGCGGGCCAA-3'), final concentration of $0.4 \mathrm{mM}, 0.3 \mu \mathrm{L}(1.5 \mathrm{U})$ of the DFS Taq DNA polymerase (BIORON, Germany), $1 \mu \mathrm{L}$ of the boiled DNA (final concentration $2 \mathrm{ng} / \mu \mathrm{L}$ ), and DNase-, RNasefree deionised water (Biomedicals) to a final volume of 25 $\mu \mathrm{L}{ }^{15}$ Amplification was carried out in a thermal cycler (Applied Biosystems, USA) as follows: the primary denaturation for 5 minutes at $95^{\circ} \mathrm{C}$; followed by 30 cycles of 60 seconds for $94^{\circ} \mathrm{C}, 60$ seconds at annealing temperature, and 5 minutes at $72^{\circ} \mathrm{C}$; and the final extension phase for 15 minutes at $72^{\circ} \mathrm{C}$. The analysis of the amplified products was performed in $1 \%$ agarose (Sigma), and DNA bands were visualized by staining with gel red (Biotium), analyzed under UV light, and photographed using the GEL Doc 2000 documentation system (Bio-Rad). Comparison of RAPD-PCR banding patterns was performed using Gelcompar II, version 6.5 (Applied Maths, SintMatensLatem, Belgium), and the unweighed pair group method with arithmetic mean (UPGMA) and dendrograms were constructed.

\section{Statistical Analysis}

Descriptive statistics were reported by total numbers, percentages, means, and medians as appropriate. 


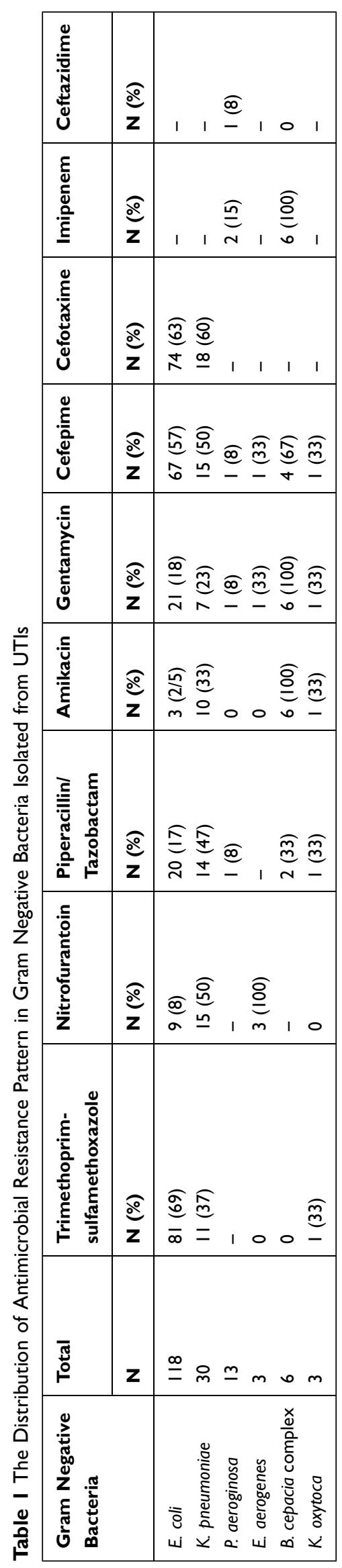

\section{Results}

A total of 203 children with positive urine cultures, collected in the tertiary referral Children's Medical Hospital in Tehran, Iran, were included in our study. Among these patients, $130(64 \%)$ subjects were female. Median age was 1 year (IQR $=4$ months to 4 years), and the age range was 1 day to 16 years.

E. coli (118 samples, 58\%) was the most prevalent microorganism isolated from urine cultures, followed by $K$. pneumoniae (30 samples, 15\%) and $P$. aeruginosa (13 samples, $6 \%$ ). Among the isolated gram positive microorganisms, the most frequent isolated bacteria were E. faecium (13 samples, $6 \%$ ) and $S$. epidermidis (5 samples, $2.5 \%$ ), respectively. The other strains were identified as: $B$. cepacia complex, E. aerogenes, K. oxytoca, E. cloacae complex, $P$. mirabilis, Morganella morganii, Acinetobacter baumanniibaumannii, P. vulgaris, Enterobacter spp., E. feacalis, S. haemolyticus, and Alpha Hemolytic Streptococcus (viridans group).

A high percentage of $E$. coli strains were isolated from female children $(81 \%)$, while $70 \%$ of $K$. pneumoniae strains were isolated from male children. Most of the bacteria were isolated from the emergency ward $(\mathrm{N}=51$, $25 \%)$, followed by the urology $(\mathrm{N}=33,16 \%)$ and nephrology $(\mathrm{N}=32,16 \%)$ wards.

The results of the antibiotic resistance pattern to the mentioned antibiotic disks for each microorganism are shown in Tables 1 and 2. Sixty percent of $K$. pneumoniae and $69 \%$ of E. coli strains were resistant to cefotaxime and trimethoprim-sulfamethoxazole, respectively. Twenty-three percent of E. faecium strains $(\mathrm{n}=3)$ showed resistance to vancomycin, which was lower than our previous reports. ${ }^{15,16}$

Out of 203 strains isolated, 62 strains had nosocomial infection criteria which were: 18 E. coli, 13 K. pneumoniae, 11 E. faecium, and five B. cepacia complex. Molecular typing and phylogenetic analysis of these 62 isolates causing hospital-acquired infection revealed that $E$. coli strains were divided into three clusters with $70 \%$ genetic similarity (Figure 1A). Also, the presence of all $K$. pneumoniae strains in one cluster with high genetic similarity was reported (Figure 1B), most of which were isolated from the Nephrology ward and Neonatal Intensive Care Unit (NICU). Among five B. cepacia complex isolates, four strains had 90\% genetic similarity (Figure 1C).

The dendrogram, based on analysis of E. faecium strains, indicated the presence of two clusters with more 


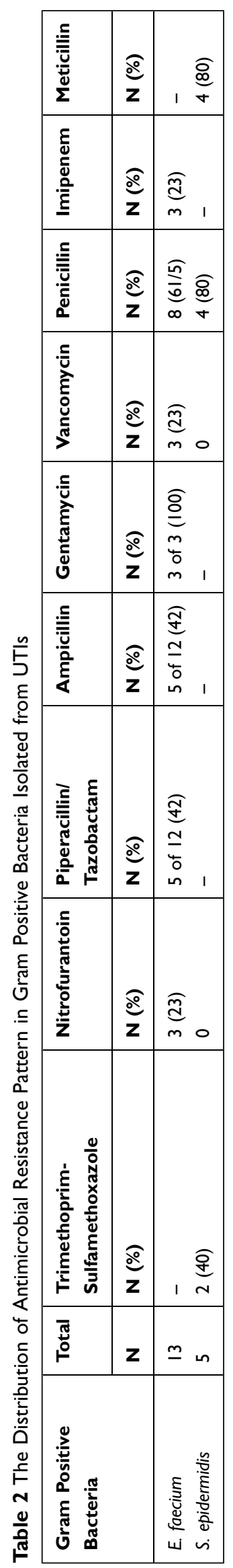

than $70 \%$ genetic similarity (Figure 1D). Two strains were completely similar to each other, isolated from different wards.

\section{Discussion}

In this study, we report the antimicrobial resistance and genotyping of bacteria isolated from UTIs in children in an Iranian referral hospital. The current concern of UTIs is the prevalence of multi drug resistance and also a main problem in the treatment of UTIs. ${ }^{17,18}$

In our study among 203 positive cultures, the most frequent pathogen was E. coli (58\%), which is generally the leading uropathogen and was isolated from $56.6-84.6 \%$ of Iranian children with febrile UTI. ${ }^{19,20}$ Similarly, it is reported as frequent as $62 \%$ in Raman et al's ${ }^{21}$ study. However, in Heidary et al study $P$. aeruginosa was reported as the most common pathogen (49.65\%).

In this study, E. coli isolates revealed a high resistance rate to trimethoprim-sulfamethoxazole (69\%) which has increased compared with the percentage of this antibiotic in previous studies. ${ }^{21}$ According to a report by Bryce et $\mathrm{al},{ }^{22}$ pooled prevalence of resistance to trimethoprim-sulfamethoxazole was as high as $30 \%$ in OECD (Organization for Economic Cooperation and Development) countries and resistance to trimethoprim-sulfamethoxazole was more than twice as high in non-OECD compared with OECD countries. On the other hand, the highest percentages of susceptibility of E. coli in our current study were reported for amikacin (97.5\%) and nitrofurantoin (91\%), which was similar to our previous study. $^{23}$

A high $P$. aeruginosa susceptibility rate to amikacin $(100 \%)$, ceftazidime, cefepime, gentamycin, piperacillin/ tazobactam (92\%), and imipenem (85\%) was reported in the current study which was higher than the frequency reported by Zhang et al. ${ }^{24}$ Genotyping of 18 E. coli strains causing nosocomial infections depicted the presence of three clusters with more than $70 \%$ genetic similarity, 12 of 18 strains were isolated from urology or surgical units. These results suggested the possibility of dissemination of these clones, especially in these two units.

More than half of the K. pneumoniae isolates were resistant to cefepime and cefotaxime. In addition, all $K$. pneumoniae isolates belonged to one cluster and most of the strains were isolated from the nephrology ward or NICU, suggesting a nosocomial infection between these two units. Similar to the current result, our previous study reported the presence of $93 \%$ of $K$. pneumoniae isolates causing nosocomial infections in one cluster. ${ }^{25}$ 
$A^{\text {UTI }}$

UTI
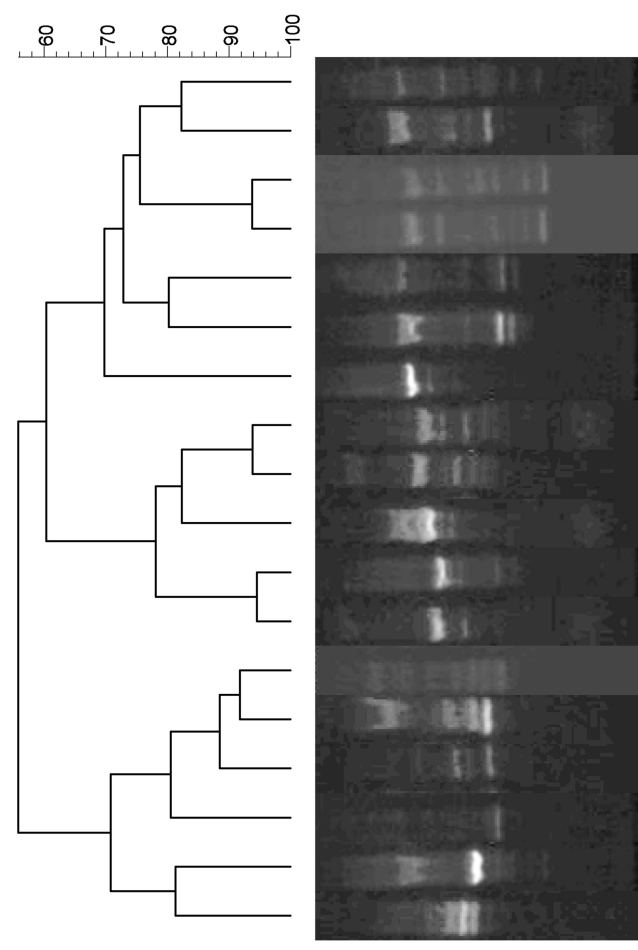

E28

E68

E136

E162

E1

E97

E38

E69

E80

E75

E147

E74

E14

E65

E63

E95

E29

E79

C

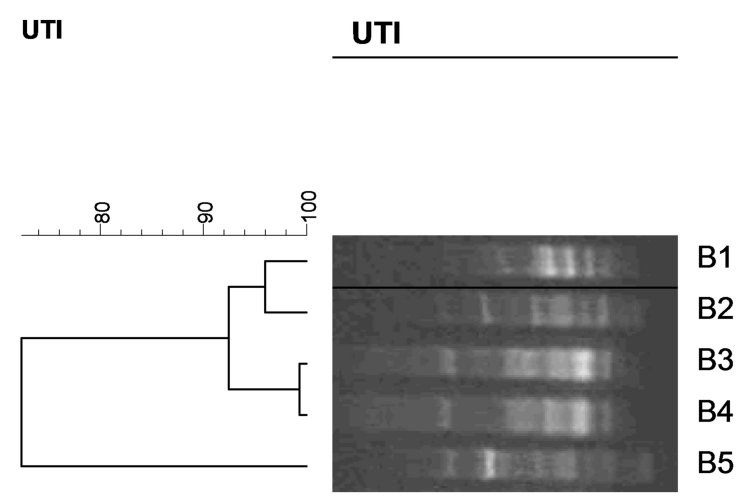

B

UTI

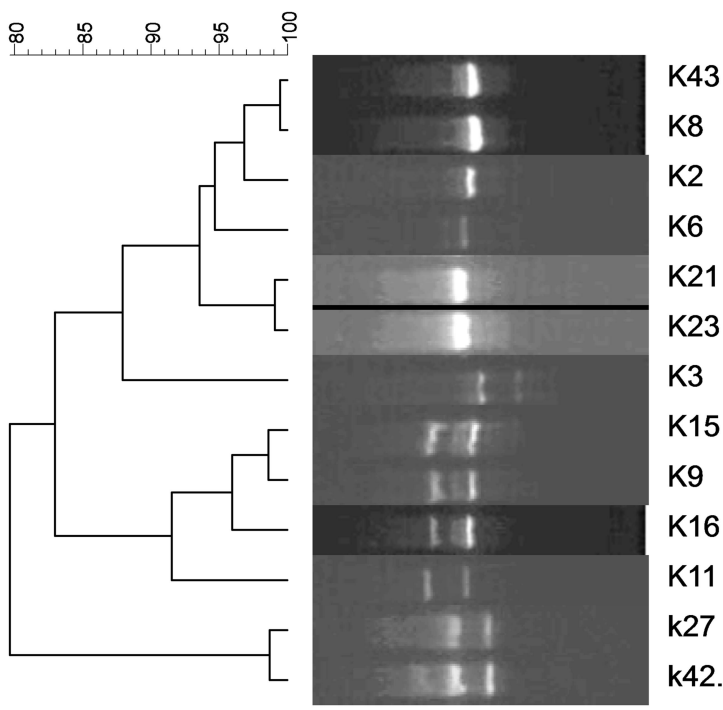

UTI

UTI

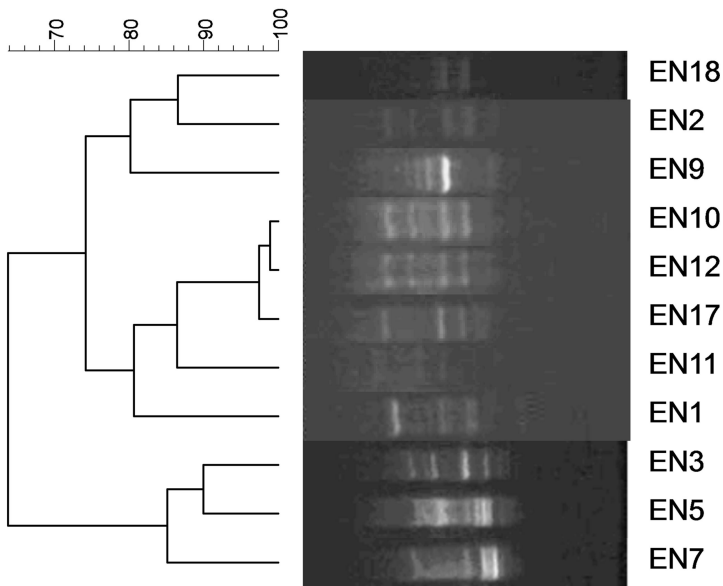

Figure I Genotyping of E. coli (A), K. pneumoniae (B), B. cepacia complex (C), and E. faecium strains (D) that met hospital-acquired infection criteria using RAPD-PCR.

Treatment of infections caused by B. cepacia complex, opportunistic nosocomial pathogens, relies on ceftazidime and other extended-spectrum cephalosporins, as intrinsic resistance prevents the action of many other classes of antimicrobials. $^{26}$ In our study, all B. cepacia complex isolates were susceptible to ceftazidime and $100 \%$ resistant to amikacin and gentamycin. In a study conducted in Taiwan, $24 \%$ of B. cepacia complex were resistant to ceftazidime and, similar to ours, had a high susceptibility to trimethoprim-sulfamethoxazole. ${ }^{27}$ The genotyping of B. cepacia complex depicted that $80 \%$ of isolates had high genetic similarity, isolated from urology, nephrology units, and NICU. Therefore, the spread of one strain between these three wards is probable.

The analysis of RAPD-typing of E. faecium strains showed the presence of two clusters with more than $70 \%$ genetic similarity and most of the strains were isolated from urology and nephrology units. 
The RAPD-PCR is a simple, rapid, easy, inexpensive, and reproducible method that has been widely used in bacterial epidemiology and finding of bacterial genetic similarity that is crucial for cross-infection assessment. ${ }^{15,25,28,31}$ Cross-sectional transmission, especially from the urology unit to other wards, may lead to strains transmission causing nosocomial infection through the hospital. Therefore, much attention should be paid to the basic methods of preventing infection (standard precautions). ${ }^{32,33}$ The length of hospitalization and the use of antibiotics in patients, particularly during longterm hospitalization and unnecessary transfer of patients between different units might have impacts on the reduction of antibiotic resistance and the occurrence of nosocomial infection.

There are several limitations for this study. First, this is a single-center retrospective study using existing data collected over years with small samples size. It is possible that we could have missed cases such as those who were treated without any culture testing, or received antibiotics before urine collection for culture. Second, there was no access to some clinical information of the patients such as the results of treatment, so determining the rate of the outcome was impossible.

In conclusion, the most frequent isolated pathogen was E. coli and the increasing rate of antibiotic resistance to the commonly prescribed antimicrobial agents such as trimetho$\mathrm{prim} / \mathrm{sulfamethoxazole} \mathrm{and} \mathrm{cephalosporins} \mathrm{was} \mathrm{observed.}$ Moreover, the results of this study showed the presence of clones with $\geq 80 \%$ similarity in E. coli, K. pneumoniae, E. faecium, and B. cepacia complex isolates; therefore, the transmission of nosocomial infections from one patient to another or one ward to another is probable.

\section{Acknowledgments}

This study was taken from Dr. Zohreh Shalchi's postgraduate thesis and was supported by a grant (number: 97-0288-39126) from Tehran University of Medical Sciences to Dr. Babak Pourakbari.

\section{Disclosure}

The authors report no conflicts of interest in this work.

\section{References}

1. Okarska-Napierała M, Wasilewska A, Kuchar E. Urinary tract infection in children: diagnosis, treatment, imaging-Comparison of current guidelines. J Pediatr Urol. 2017;13(6):567-573. doi:10.1016/j. jpurol.2017.07.018
2. Kutasy B, Coyle D, Fossum M. Urinary tract infection in children: management in the era of antibiotic resistance - a pediatric urologist's view. Eur Urol Focus. 2017;3(2-3):207-211. doi:10.1016/j. euf.2017.09.013

3. Wang J, He L, Sha J, et al. Etiology and antimicrobial resistance patterns in pediatric urinary tract infection. Pediatrics Int. 2018;60 (5):418-422. doi:10.1111/ped.13526

4. Awean GZ, Salameh K, Elmohamed H, Alshmayt H, Omer MRB. Prevalence of ESBL urinary tract infection in children. $J$ Adv Pediatr Child Health. 2019;2(1):004-7. doi:10.29328/journal.japch.1001004

5. Pouladfar G, Basiratnia M, Anvarinejad M, Abbasi P, Amirmoezi F, Zare S. The antibiotic susceptibility patterns of uropathogens among children with urinary tract infection in Shiraz. Medicine. 2017;96 (37):37. doi:10.1097/MD.0000000000007834

6. Dahle KW, Korgenski EK, Hersh AL, Srivastava R, Gesteland PH. Clinical value of an ambulatory-based antibiogram for uropathogens in children. $J$ Pediatric Infect Dis Soc. 2012;1(4):333-336. doi:10.1093/jpids/pis055

7. Zorc JJ, Kiddoo DA, Shaw KN. Diagnosis and management of pediatric urinary tract infections. Clin Microbiol Rev. 2005;18 (2):417-422. doi:10.1128/CMR.18.2.417-422.2005

8. Amraei S, Hashemi Karouei SM, Babakhani S, Kazemi MJ. Serotyping and antibiotic susceptibility pattern of common bacterial uropathogens in urinary tract infections in Koohdasht, Lorestan province. Int J Enteric Pathog. 2016;4(2):1-5.

9. Nor NSM, Abu NA, Rashid MA, Ismail M, Razak R. Bacterial pathogens and antibiotic resistance patterns in children with urinary tract infection in a Malaysian tertiary hospital. Med J Malays. 2015;70(3):153-157.

10. Doyev R, Ben-Shalom E, Megged O. The predictive utility of prior positive urine culture in children with recurrent urinary tract infections. Eur J Pediatr. 2020;179(3):415-421. doi:10.1007/s00431-019-03522-5

11. Koçak M, Büyükkaragöz B, Çelebi Tayfur A, et al. Causative pathogens and antibiotic resistance in children hospitalized for urinary tract infection. Pediatrics Int. 2016;58(6):467-471. doi:10.1111/ped.12842

12. Prais D, Straussberg R, Avitzur Y, Nussinovitch M, Harel L, Amir J. Bacterial susceptibility to oral antibiotics in community acquired urinary tract infection. Arch Dis Child. 2003;88(3):215-218. doi:10.1136/adc.88.3.215

13. Mahon CR, Lehman DC, Manuselis G. Textbook of Diagnostic Microbiology 2007. 2008. 3rd ed. St. Louis, Mo: Saunders Elsevier.

14. <melvin p. performance standards for antimicrobial susceptibility testing. 2019.pdf>

15. Mamishi S, Mohammadian M, Pourakbari B, et al. Antibiotic resistance and genotyping of gram-positive bacteria causing hospitalacquired infection in patients referring to children's medical center. Infect Drug Resist. 2019;12:3719-3726. doi:10.2147/IDR.S220522

16. Pourakbari B, Mahmoudi S, Moradzadeh M, et al. Antimicrobial Resistance Patterns of the Gram-positive Bacteria Isolated from Children with Bloodstream Infection in an Iranian Referral Hospital: A 6-year Study. Infect Disord Drug Targets. 2018;18 (2):136-144. doi:10.2174/1871526517666170821164343

17. Niranjan V, Malini A, Bahaman AR, Amran F, Khalilpour A. Antimicrobial resistance pattern in Escherichia coli causing urinary tract infection among inpatients. Indian J Med Res. 2014;139(6):945.

18. Marialouis XA, Santhanam A. Antibiotic resistance, RAPD-PCR typing of multiple drug resistant strains of Escherichia coli from urinary tract infection (UTI). J Clin Diagn Res. 2016;10(3):DC05.

19. Afsharpaiman S, Bairaghdar F, Torkaman M, et al. Bacterial pathogens and resistance patterns in children with community-acquired urinary tract infection: a cross sectional study. $J$ Comprehens Pediatr. 2012;3(1):16-20. doi:10.5812/jcp.7078

20. Mirsoleymani SR, Salimi M, Shareghi Brojeni M, Ranjbar M, Mehtarpoor M. Bacterial pathogens and antimicrobial resistance patterns in pediatric urinary tract infections: a four-year surveillance study (2009-2012). Int J Pediatr. 2014;2014. 
21. Raman G, McMullan B, Taylor P, Mallitt K-A, Kennedy SE, Multiresistant E. coli urine infections in children: a case-control study. Arch Dis Child. 2018;103(4):336-340. doi:10.1136/archdischild-2017-312831

22. Bryce A, Hay AD, Lane IF, Thornton HV, Wootton M, Costelloe C. Global prevalence of antibiotic resistance in paediatric urinary tract infections caused by Escherichia coli and association with routine use of antibiotics in primary care: systematic review and meta-analysis. BMJ. 2016;352:i939. doi:10.1136/bmj.i939

23. Pourakbari B, Ferdosian F, Mahmoudi S, et al. Increase resistant rates and ESBL production between $\mathrm{E}$. coli isolates causing urinary tract infection in young patients from Iran. $\mathrm{Br} J$ Microbiol. 2012;43 (2):766-769. doi:10.1590/S1517-83822012000200041

24. Zhang X, Niu S, Zhang L. Antimicrobial susceptibilities and clinical characterization of Pseudomonas aeruginosa isolates from urinary tract infections. Urol Int. 2014;93(4):464-469. doi:10.1159/ 000358493

25. Mahmoudi S, Pourakbari B, Rahbarimanesh A, Abdosalehi MR, Ghadiri K, Mamishi S. An outbreak of ESBL-producing Klebsiella pneumoniae in an Iranian referral hospital: epidemiology and molecular typing. Infect Dis Drug Targets. 2019;19(1):46-54.

26. Rhodes KA, Schweizer HP. Antibiotic resistance in Burkholderia species. Drug Resist Updates. 2016;28:82-90. doi:10.1016/j. drup.2016.07.003

27. Tseng S-P, Tsai W-C, Liang C-Y, et al. The contribution of antibiotic resistance mechanisms in clinical Burkholderia cepacia complex isolates: an emphasis on efflux pump activity. PLoS One. 2014;9(8):8. doi:10.1371/journal.pone.0104986
28. Baker JC, Crumley RE, Eckdahl TT. Random amplified polymorphic DNA PCR in the microbiology teaching laboratory: identification of bacterial unknowns. Biochem Mol Biol Educ. 2002;30(6):394-397. doi:10.1002/bmb.2002.494030060135

29. Pourakbari B, Mahmoudi S, Aghdam MK, et al. Clonal spread of vancomycin resistance Enterococcus faecalis in an Iranian referral pediatrics center. J Prev Med Hyg. 2013;54(2):87-89.

30. Pourakbari B, Mahmoudi S, Habibi R, et al. An increasing threat in an Iranian referral children's hospital: multidrug-resistant acinetobacter baumannii. Infect Disord Drug Targets. 2018;18(2):129-135. doi:10.2174/1871526517666170113144541

31. Hassuna NA, Darwish MK, Sayed M, Ibrahem RA. Molecular epidemiology and mechanisms of high-level resistance to meropenem and imipenem in pseudomonas aeruginosa. Infect Drug Resist. 2020;13:285. doi:10.2147/IDR.S233808

32. Mamishi S, Pourakbari B, Teymuri M, Babamahmoodi A, Mahmoudi S. Management of hospital infection control in iran: a need for implementation of multidisciplinary approach. Osong Public Health Res Perspect. 2014;5(4):179-186. doi:10.1016/j.phrp.2014.06.001

33. Movahedi Z, Pourakbari B, Mahmoudi S, et al. Pseudomonas aeruginosa infection among cystic fibrosis and ICU patients in the referral children medical hospital in Tehran, Iran. J Prev Med Hyg. 2013;54(1):24.
Infection and Drug Resistance

\section{Publish your work in this journal}

Infection and Drug Resistance is an international, peer-reviewed openaccess journal that focuses on the optimal treatment of infection (bacterial, fungal and viral) and the development and institution of preventive strategies to minimize the development and spread of resistance. The journal is specifically concerned with the epidemiology of antibiotic resistance and the mechanisms of resistance development and diffusion in both hospitals and the community. The manuscript management system is completely online and includes a very quick and fair peerreview system, which is all easy to use. Visit http://www.dovepress.com/ testimonials.php to read real quotes from published authors. 